\title{
A statistical framework for selecting natural fibre reinforced polymer composites based on regression model
}

\begin{abstract}
Material selection is an important stage in the development of products from composites process of automotive component application. Numerious different Multi-Criteria DecisionMaking tools have their own strenghts and limitations. This paper presents a framework for material selection of natural fibre reinforced polymer composites by using statistical approach. The framework is developed using statistical methods which are simple, multiple and stepwise regression for the material selection process. The performance of potential material is investigated by a statistical analysis such as coefficient of correlation, coefficient of determination and analysis of variance. A case study to select the best composite of parking brake lever is applied to this framework. End results revealed that kenaf reinforced polypropylene is the best candidate for construction of automotive parking brake lever component. The best possible of statistical model for material selection of the composite can be referred by design engineer in composite industry for a multiple application. Moreover, the proposed framework is an aid to help engineers and designers to choose most suitable material.
\end{abstract}

Keyword: Material selection; Simple linear regression; Multiple linear regression; Stepwise regression; Natural fibre reinforced composites 